\title{
Computational homogenization of sound propagation in a deformable porous material including microscopic viscous- thermal effects
}

\author{
Citation for published version (APA): \\ Gao, K., van Dommelen, J. A. W., Göransson, P., \& Geers, M. G. D. (2016). Computational homogenization of \\ sound propagation in a deformable porous material including microscopic viscous-thermal effects. Journal of \\ Sound and Vibration, 365, 119-133. https://doi.org/10.1016/j.jsv.2015.11.037
}

\section{Document license: \\ TAVERNE}

DOI:

10.1016/j.jsv.2015.11.037

Document status and date:

Published: 17/03/2016

\section{Document Version:}

Publisher's PDF, also known as Version of Record (includes final page, issue and volume numbers)

\section{Please check the document version of this publication:}

- A submitted manuscript is the version of the article upon submission and before peer-review. There can be important differences between the submitted version and the official published version of record. People interested in the research are advised to contact the author for the final version of the publication, or visit the DOI to the publisher's website.

- The final author version and the galley proof are versions of the publication after peer review.

- The final published version features the final layout of the paper including the volume, issue and page numbers.

Link to publication

\footnotetext{
General rights

- You may freely distribute the URL identifying the publication in the public portal. follow below link for the End User Agreement:

www.tue.nl/taverne

\section{Take down policy}

If you believe that this document breaches copyright please contact us at:

openaccess@tue.nl

providing details and we will investigate your claim.
}

Copyright and moral rights for the publications made accessible in the public portal are retained by the authors and/or other copyright owners and it is a condition of accessing publications that users recognise and abide by the legal requirements associated with these rights.

- Users may download and print one copy of any publication from the public portal for the purpose of private study or research.

- You may not further distribute the material or use it for any profit-making activity or commercial gain

If the publication is distributed under the terms of Article $25 \mathrm{fa}$ of the Dutch Copyright Act, indicated by the "Taverne" license above, please 


\title{
Computational homogenization of sound propagation in a deformable porous material including microscopic viscous-thermal effects
}

\author{
K. Gao ${ }^{\text {a }}$, J.A.W. van Dommelen ${ }^{\text {a,*, }}$ P. Göransson ${ }^{\text {b }}$, M.G.D. Geers ${ }^{\text {a }}$ \\ a Mechanics of Materials, Department of Mechanical Engineering, Eindhoven University of Technology, 5600 MB Eindhoven, The \\ Netherlands \\ ${ }^{\mathrm{b}}$ Marcus Wallenberg Laboratory, Department of Aeronautical and Vehicle Engineering, KTH Royal Institute of Technology, SE-100 44 \\ Stockholm, Sweden
}

\section{A R T I C L E I N F O}

\section{Article history:}

Received 21 May 2015

Received in revised form

12 October 2015

Accepted 27 November 2015

Handling Editor: D. Juve

Available online 18 December 2015

Keywords:

Acoustic;

Poroelastic;

Homogenization;

Multiscale

\begin{abstract}
A B S T R A C T
Porous materials like acoustic foams can be used for acoustic shielding, which is important for high-tech systems and human comfort. In this paper, a homogenization model is proposed to investigate the relation between the microstructure and the resulting macroscopic acoustic properties. The macroscopic absorption ability is due to the microscopic viscousthermal coupling between an elastic solid skeleton and a gaseous fluid in the associated Representative Volume Element (RVE). The macro-to-micro relation is realized through the boundary conditions of the microscopic RVE, which relies on the macroscopic solid deformation and fluid pressure gradient. By assuming that the variation of the macroscopic energy per unit volume equals the volume average of the variation of the microscopic energy, the macroscopic solid stress and fluid displacement can be calculated from the corresponding microscopic quantities. Making additional assumptions on this approach, Biot's poroelastic theory is recovered. A case study is performed through the simulations of sound absorption in three porous materials, one made from aluminum and two from different polyurethane foams. For simplicity, an idealized partially open cubic microstructure is adopted. The homogenization results are evaluated by comparison with Direct Numerical Simulations (DNS), revealing an adequate performance of the approach for the studied porous material. By comparing the results of different solid materials, it is found that the solid stiffness has a limited effect when resonance does not occur. Nevertheless, due to the absence of the microscopic fluctuation, Biot's model with the parameters obtained from the homogenization approach predicts a higher resonance frequency than the DNS, whereas a full homogenization modification improves the prediction.
\end{abstract}

(c) 2015 Elsevier Ltd. All rights reserved.

\section{Introduction}

Passive sound absorbing porous materials, such as acoustic foams, can be used in acoustic shielding applications to improve the sound absorption performance. Models of acoustic porous materials can be classified as equivalent fluid models

\footnotetext{
* Corresponding author.

E-mail address: J.A.W.v.Dommelen@tue.nl (J.A.W. van Dommelen).
} 
for motionless solid skeletons or fluid-solid coupled models. In the equivalent fluid model, acoustic properties depend on the effective density and the effective bulk modulus of the fluid in the porous material. Several models of this type have been proposed. For example, Zwikker and Kosten derived an analytical model from studying the case of a cylindrical pore, where viscous and thermal effects are both considered [1]. Another widely-accepted semi-phenomenological model is the Johnson-Champoux-Allard-Lafarge (JCAL) model which combines the Johnson-Koplik-Dashen (JKD) model and the Champoux-Allard-Lafarge (CAL) model. By introducing the concept of a viscous characteristic length, the JKD model gives an analytical expression matching the low- and high-frequency limiting effective density involving the static permeability and the tortuosity at infinite frequency [2]. Analogously, Champoux and Allard introduced a thermal characteristic length and derived an analytical expression for the effective bulk modulus by assuming circular-cylindrical pores [3]. The Champoux-Allard model was further developed for more general pores by introducing the static thermal permeability [4]. Conventionally, the above non-acoustical parameters are obtained from experiments [5-8], showing satisfactory results for wool materials [9] and fibrous materials [10]. Besides, an idealized unit cell representing the microstructure of the porous material can be constructed based on geometrical parameters obtained from microstructure characterization techniques [11-14]. The non-acoustical parameters can be linked to the representative unit cell through these semi-phenomenological models using (semi-)empirical relations [13] or calculated numerically by solving a steady Stokes problem, an electric conduction problem and a thermal conduction problem of the unit cell $[11,12]$. Justifications of these computational schemes can be obtained, clarified and developed by applying the asymptotic homogenization method to the fluid domain in the porous material [15-18]. Following an electromagnetic-acoustic analogy and an ensemble-averaging concept, a nonperturbative homogenization method was developed to allow for both temporal and spatial dispersion effects in fluidsaturated rigid-framed porous media [19].

A limitation of the equivalent fluid models is the lack of solid motion, which is important in vibro-acoustic problems [20] and which may be added as a refinement. Furthermore, although the sound absorbing behavior is often believed to be mainly governed by the local visco-thermal dissipations of the fluid, in particular for some partially-reticulated foams, the vibration of the pore membranes is observed to have a significant influence [21] and the consideration of the solid elastic properties can improve the agreement with experimental measurements [22]. Therefore, a fluid-solid coupling model should be considered when the solid motion is not negligible. The most famous coupling model is probably Biot's model, based on Biot's poroelastic theory [23-26], describing the coupling between the macroscopic fluid and solid displacement fields with effective parameters that are dependent on the corresponding microstructure. It can be easily expanded for anisotropic materials [27,28] and implemented in a finite element context [29-33]. Biot's model includes the effects of the microstructure implicitly through the effective parameters: the viscous coefficient and the added density are mainly determined by the effective fluid density; the elastic coefficients are related to the effective bulk modulus of the solid skeleton and the fluid through three gedanken experiments for isotropic porous materials [34]. A widely accepted method for obtaining the effective fluid properties involved in Biot's model is a direct application of equivalent fluid models such as the JCAL model [35-39]. There are also other analytical models to obtain Biot's parameters, such as Pride et al.'s model [40] and Wilmanski's model [41]. On the other hand, many fluid-solid coupling models have also been studied by taking into account the microstructure explicitly. For example, the asymptotic homogenization method has been applied to a porous material including an elastic solid skeleton and a compressible viscous gaseous fluid, each with a linearized behavior [4245,15]. By considering the porous material as a mixture of a solid and a fluid, a set of macroscopic thermodynamically consistent constitutive equations can be obtained, while applying volume integration on the microscopic mass and momentum conservation equations [46-48].

Recently, the authors proposed a multiscale homogenization approach to obtain Biot's parameters from the corresponding microstructure by adopting the computational homogenization framework [49]. The proposed homogenization method is based on the scale separation principle that is also a fundamental assumption in the asymptotic homogenization method. However, it does not require lots of mathematical derivations and assesses the macroscopic influence of the complex microstructure straightforwardly. Furthermore, instead of a direct volume-averaging [40,46-48] or an ensembleaveraging [19], the upscaling from the microscopic scale to the macroscopic scale is realized through energy consistency of the two scales in the proposed method. It gives a good description of the viscous fluid-solid coupling effect in porous materials under isothermal conditions for the microscopic RVE. However, to agree with Biot's theory, the microscopic solid fluctuations were ignored in the approach, which may lead to an inaccurate description of the macroscopic properties. Moreover, the thermal effect is also very important for acoustic porous materials, particularly at low frequencies [1,3,4].

In this paper, extensions of the homogenization approach are proposed at both the macroscopic and the microscopic scales. Instead of Biot's poroelastic theory, the general formulations of momentum conservation of the solid and mass conservation of the fluid are adopted for the description of the macroscopic problem. Considering the linearity of the problem, an enhanced homogenization is obtained and it is compatible with Biot's poroelastic theory only when the microscopic fluctuations of the solid and the average of the microscopic fluid dilation are negligible. Through numerical examples of a simple unit cell with several different solid materials, it is shown that the incorporation of the microscopic fluctuations is crucial for a correct description of the effective solid density. A comparison with the results of Direct Numerical Simulation (DNS) shows that this new model gives a more accurate result when resonance occurs. Besides, in the microscopic RVE, the non-uniform thermal field is considered by applying the set of linearized Navier-Stokes-Fourier equations in the fluid [50] and allowing the thermal diffusion in the solid. The classical description of the non-isothermal 
field in the microscopic RVE has been widely applied for porous materials $[1,4,45,19]$ and it is an essential extension of the proposed homogenization method, giving a more reasonable sound absorption performance at low frequencies.

\section{Homogenization framework}

The objective of this section is to present a homogenization framework for a general acoustic problem in porous materials. In this paper, the problem is studied in the frequency domain and the time derivative $\partial / \partial t$ is replaced by j $\omega$ with $\omega$ being the angular frequency and $\mathrm{j}$ the imaginary unit. The macroscopic quantities are indicated by the subscript $M$ and the microscopic ones are denoted by the subscript $m$. The double dot product between two 2nd-order tensors $\boldsymbol{a b}$ (a dyadic product of vectors $\boldsymbol{a}$ and $\boldsymbol{b}$ ) and $\boldsymbol{c d}$ (a dyadic product of vectors $\boldsymbol{c}$ and $\boldsymbol{d}$ ) is defined as

$$
\boldsymbol{a b}: \boldsymbol{c d}=(\boldsymbol{a} \cdot \boldsymbol{d})(\boldsymbol{b} \cdot \boldsymbol{c}) .
$$

The fundamental assumption in the homogenization method is the scale separation principle, which requires that the macroscopic characteristic length $L_{M}^{*}$ is much larger than the microscopic characteristic length $R$. In this case, it is possible to separate the multiscale problem for porous materials into a macroscopic problem and a microscopic one. Furthermore, the microscopic characteristic length is assumed to be large enough to ensure that continuum mechanics is valid at the microscopic scale. Taking $R$ as the characteristic pore size for porous materials, the acoustic wavelength $\lambda$ should not be smaller than the macroscopic characteristic length. Together, the scale separation requirement in the problem leads to

$$
\lambda \geq L_{M}^{*} \gg R \text {. }
$$

For common acoustic porous materials, $R$ is about $10^{-4} \sim 10^{-3} \mathrm{~m}$. To ensure Eq. (2) is satisfied, the shortest wavelength should be at least $\mathrm{O}\left(10^{-1}\right) \mathrm{m}$. Therefore, the maximum frequency studied in this paper is restricted to $5000 \mathrm{~Hz}$. The lowest frequency studied in this paper is set as $100 \mathrm{~Hz}$ without loss of generality.

\subsection{Macroscopic problem}

In the macroscopic problem, two coupled phases are considered: a solid skeleton and a gaseous fluid. To describe the deformability of the solid, the solid configuration in the static undeformed state is chosen as the initial configuration denoted by $\boldsymbol{x}_{0 M}^{S}$ and the current configuration is denoted by $\boldsymbol{x}_{M}^{S}$. In this paper, a Lagrangian description is adopted. Assuming that the deformations are small, the current configuration and the initial configuration are almost identical. The porosity $\phi$, defined as the volume fraction of the fluid, remains constant in this case. The density variations of the solid and the fluid are ignored, since they are much smaller than the static densities $\rho_{0}^{s}$ and $\rho_{0}^{f}$ within the small deformation assumption. Given the small deformation assumption and the low viscosity of a common gaseous fluid (the viscosity of air is around $10^{-5}$ Pa $\mathrm{s}$ ), the macroscopic viscous stress in the fluid is negligible compared to the macroscopic pressure and is therefore ignored in the stress-strain relation. Nevertheless, the viscous dissipation is still accounted for through the permeability. In this case, the fluid flow is a potential flow in which the potential is determined from the fluid pressure $P_{M}^{f}$ and the solid displacement $\boldsymbol{u}_{M}^{s}$. On the other hand, due to the low thermal diffusivity (the thermal diffusivity of air is around $2 \times 10^{-5} \mathrm{~m}^{2} / \mathrm{s}$ ), the macroscopic work due to thermal diffusion of the fluid can be ignored compared to the macroscopic pressure work. Besides, by assuming that there is no large temperature gradient and no heat source in the porous material, the macroscopic work due to thermal diffusion in the solid is much smaller than the macroscopic mechanical work performed on the solid because of the small temperature variations induced by the acoustic wave (in the order of $10^{-3} \sim 10^{-1} \mathrm{~K}$ when the sound pressure level ranges from $100 \mathrm{~dB}$ to $140 \mathrm{~dB}$ ). Since the macroscopic thermal diffusion can be ignored in both the fluid and the solid, the sound propagation in the porous material is considered as an adiabatic process at the macroscopic scale. Consequently, the solid displacement $\boldsymbol{u}_{M}^{s}$ and the fluid pressure $P_{M}^{f}$ are the governing macroscopic field variables.

The macroscopic governing equations are given by

$$
\boldsymbol{f}_{M}^{s}=\nabla_{M} \cdot\left(\sigma_{M}^{s}\right)^{\mathrm{T}}, \text { and } \epsilon_{M}^{f}-\nabla_{M} \cdot \boldsymbol{u}_{M}^{f}=0 .
$$

Here the operator $\nabla_{M}$ represents the spatial gradient at the macroscopic scale. The first equation in Eq. (3) represents the momentum conservation of the solid, where $\boldsymbol{\sigma}_{M}^{s}$ is the macroscopic Cauchy stress of the solid and $\boldsymbol{f}_{M}^{s}$ is the inertial force exerted on the solid. The second equation represents the mass conservation of the fluid, where $\epsilon_{M}^{f}$ is the macroscopic volumetric change of the fluid and $\boldsymbol{u}_{M}^{f}$ is the fluid displacement. The following step is to derive the macroscopic energy variation in terms of the macroscopic field variables. Based on energy conservation and the adiabatic assumption, the macroscopic energy variation per unit volume of a macroscopic point equals the external mechanical work applied on this point. By following the same method in [49], the result is given as

$$
\delta E_{M}=(1-\phi)\left(\nabla_{M} \cdot\left(\sigma_{M}^{s}\right)^{T}\right) \cdot \delta \boldsymbol{u}_{M}^{s}+(1-\phi) \sigma_{M}^{s}: \delta \nabla_{M} \boldsymbol{u}_{M}^{s}-\phi \boldsymbol{u}_{M}^{f} \cdot \delta\left(\nabla_{M} p_{M}^{f}\right)-\phi\left(\nabla_{M} \cdot \boldsymbol{u}_{M}^{f}\right) \delta p_{M}^{f} .
$$

Here the pressure difference is $p_{M}^{f}=P_{M}^{f}-P_{0}$ with $P_{0}$ being the constant ambient pressure. According to Eq. (3), Eq. (4) is rewritten as

$$
\delta E_{M}=(1-\phi) \boldsymbol{\sigma}_{M}^{s}: \delta\left(\nabla_{M} \boldsymbol{u}_{M}^{s}\right)-\phi \epsilon_{M}^{f} \delta p_{M}^{f}+(1-\phi) \boldsymbol{f}_{M}^{s} \cdot \delta \boldsymbol{u}_{M}^{s}-\phi \boldsymbol{u}_{M}^{f} \cdot \delta\left(\nabla_{M} p_{M}^{f}\right)
$$


In this expression, the first two terms involving the macroscopic solid deformation and the macroscopic fluid dilatation together represent the variation of the potential energy per unit volume. The last two terms represent the variations of the kinetic energy and the dissipation.

\subsection{Microscopic boundary value problem}

A microscopic RVE under isothermal conditions has been discussed in [49]. This paper focuses on the implementation of a non-uniform thermal field. To understand the derivation easily, the following discussion first focuses on the time domain and the result will be converted to the frequency domain by replacing the time derivative $\partial / \partial t$ with j $\omega$ afterwards. For linear acoustic problems, with small deformations, the microscopic pressure work is much more significant than the microscopic viscous dissipation for air when the frequency is lower than $10^{4} \mathrm{~Hz}$. Hence, by ignoring the viscous dissipation term, energy conservation of the fluid gives [50]

$$
\rho_{m}^{f} \frac{\partial}{\partial t}\left(C_{p}^{f} T_{m}^{f}-\frac{P_{m}^{f}}{\rho_{m}^{f}}\right)=-P_{m}^{f} \nabla_{m} \cdot \dot{\boldsymbol{u}}_{m}^{f}-\nabla_{m} \cdot \boldsymbol{q}_{m}^{f},
$$

where $\rho_{m}^{f}$ is the fluid density, $C_{p}^{f}$ is the thermal capacity at constant pressure, $T_{m}^{f}$ is the temperature, $P_{m}^{f}$ is the pressure, $\boldsymbol{u}_{m}^{f}$ is the fluid displacement, $\boldsymbol{\tau}_{m}^{f}$ is the viscous stress and $\boldsymbol{q}_{m}^{f}$ is the thermal flux. Here $\boldsymbol{u}$ stands for the velocity. The left hand side of Eq. (6) represents the rate of change of the internal energy of an ideal gas per unit volume and it can be further written as

$$
\rho_{m}^{f} \frac{\partial}{\partial t}\left(C_{p}^{f} T_{m}^{f}-\frac{P_{m}^{f}}{\rho_{m}^{f}}\right)=\rho_{m}^{f} C_{p}^{f} \frac{\partial T_{m}^{f}}{\partial t}-\left[\frac{\partial P_{m}^{f}}{\partial t}+\frac{P_{m}^{f}}{\rho_{m}^{f}} \nabla_{m} \cdot\left(\rho_{m}^{f} \dot{\boldsymbol{u}}_{m}^{f}\right)\right],
$$

based on mass conservation of the fluid

$$
\frac{\partial \rho_{m}^{f}}{\partial t}+\nabla_{m} \cdot\left(\rho_{m}^{f} \dot{\boldsymbol{u}}_{m}^{f}\right)=0 .
$$

Substituting Eq. (7) into Eq. (6) and ignoring the variation of the fluid density gives

$$
\rho_{0}^{f} C_{p}^{f} \frac{\partial T_{m}^{f}}{\partial t}=\frac{\partial P_{m}^{f}}{\partial t}-\nabla_{m} \cdot \boldsymbol{q}_{m}^{f}
$$

with $\rho_{0}^{f}$ being the static density. Introducing the temperature difference $\theta_{m}^{f}=T_{m}^{f}-T_{0}$ and the pressure difference $p_{m}^{f}=P_{m}^{f}-P_{0}$, the above equation is rewritten as

$$
\rho_{0}^{f} C_{p}^{f} \frac{\partial \theta_{m}^{f}}{\partial t}=\frac{\partial p_{m}^{f}}{\partial t}-\nabla_{m} \cdot \boldsymbol{q}_{m}^{f} .
$$

Here $T_{0}$ is the ambient temperature, which is constant. By assuming that $\theta_{m}^{f}$ and $p_{m}^{f}$ are harmonic with respect to the time, Eq. (10) is converted to the frequency domain:

$$
\rho_{0}^{f} C_{p}^{f} \mathrm{j} \omega \theta_{m}^{f}=\mathrm{j} \omega p_{m}^{f}-\nabla_{m} \cdot \boldsymbol{q}_{m}^{f},
$$

To relate the pressure, the density and the temperature, the ideal gas law is used:

$$
\frac{P_{0}+p_{m}^{f}}{\left(\rho_{0}^{f}+\delta_{m}^{f}\right)\left(T_{0}+\theta_{m}^{f}\right)}=\frac{P_{0}}{\rho_{0}^{f} T_{0}},
$$

with the density difference $\delta_{m}^{f}=\rho_{m}^{f}-\rho_{0}^{f}$. The ideal gas law can be rewritten as

$$
\frac{p_{m}^{f}}{P_{0}}=\frac{\theta_{m}^{f}}{T_{0}}+\frac{\delta_{m}^{f}}{\rho_{0}^{f}}+\frac{\delta_{m}^{f} \theta_{m}^{f}}{\rho_{0}^{f} T_{0}}
$$

By ignoring the last term (a second-order infinitesimal) and using a linearized mass conservation:

$$
\frac{\delta_{m}^{f}}{\rho_{0}^{f}}+\nabla_{m} \cdot \boldsymbol{u}_{m}^{f}=0
$$

the state equation is simplified into

$$
\frac{p_{m}^{f}}{P_{0}}=\frac{\theta_{m}^{f}}{T_{0}}-\nabla_{m} \cdot \boldsymbol{u}_{m}^{f} .
$$

For the solid, the thermal expansion is ignored because of the small temperature variation and the linear isotropic elastic constitutive law of the isothermal case can be directly adopted [49]. The temperature difference in the solid $\theta_{m}^{s}$ is only governed by thermal diffusion:

$$
\rho_{0}^{s} C_{p}^{s} \mathrm{j} \omega \theta_{m}^{s}=-\nabla_{m} \cdot \boldsymbol{q}_{m}^{s},
$$

where $C_{p}^{s}$ is the thermal capacity of the solid at constant pressure and $\boldsymbol{q}_{m}^{s}$ is the thermal flux. The thermal flux of the fluid 


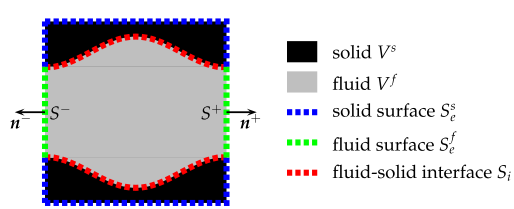

Fig. 1. Illustration of the microscopic RVE.

and the solid are both characterized by Fourier's law, i.e.

$$
\boldsymbol{q}_{m}^{f}=-k^{f} \nabla_{m} \theta_{m}^{f} \quad \text { and } \quad \boldsymbol{q}_{m}^{s}=-k^{s} \nabla_{m} \theta_{m}^{s}
$$

with $k^{f}$ and $k^{s}$ being the thermal conductivity of the fluid and the solid respectively.

Analogous to the boundary conditions used under the isothermal condition in [49], as illustrated in Fig. 1, a periodic boundary condition for the solid displacement is applied on the solid surface $S_{e}^{s}$ (where $\tilde{\boldsymbol{u}}_{m}^{s}$ is zero for the corner points):

$$
\boldsymbol{u}_{m}^{s}=\boldsymbol{u}_{M}^{s}+\left(\nabla_{M} \boldsymbol{u}_{M}^{s}\right)^{\mathrm{T}} \cdot \boldsymbol{x}_{m}^{s}+\tilde{\boldsymbol{u}}_{m}^{s} \quad \text { with } \tilde{\boldsymbol{u}}_{m}^{s+}=\tilde{\boldsymbol{u}}_{m}^{s-}, \boldsymbol{x}_{m}^{s} \in S_{e}^{s}
$$

and a prescribed traction is defined on the fluid surface $S_{e}^{f}$ by ignoring the viscous stress:

$$
\boldsymbol{n} \cdot \boldsymbol{\sigma}_{m}^{f}=-p_{m}^{f} \boldsymbol{n} \text { with } p_{m}^{f}=p_{M}^{f}+\nabla_{M} p_{M}^{f} \cdot \boldsymbol{x}_{m}^{f}, \boldsymbol{x}_{m}^{f} \in S_{e}^{f} .
$$

Here $\boldsymbol{n}$ is the outward unit normal vector on the surface; $p_{M}^{f}$ is the macroscopic pressure difference and $\tilde{\boldsymbol{u}}_{m}^{s}$ is the microscopic solid displacement fluctuation. The variables $\tilde{\boldsymbol{u}}_{m}^{s+}$ and $\tilde{\boldsymbol{u}}_{m}^{s-}$ reflect the microscopic fluctuations on two opposite boundaries $S^{+}$and $S^{-}$, which are geometrically equal in a periodic RVE. A fully continuous condition on the fluid-solid interface is adopted, i.e.,

$$
\boldsymbol{u}_{m}^{s}=\boldsymbol{u}_{m}^{f}, \quad \boldsymbol{n}_{i} \cdot \boldsymbol{\sigma}_{m}^{s}=\boldsymbol{n}_{i} \cdot \boldsymbol{\sigma}_{m}^{f} \quad \text { on } S_{i},
$$

where $\boldsymbol{n}_{i}$ is the unit normal vector on the interface and it is pointing from the fluid to the solid. Besides, the temperature and the thermal flux are also continuous on the interface

$$
\theta_{m}^{s}=\theta_{m}^{f}, \quad \boldsymbol{q}_{m}^{s} \cdot \boldsymbol{n}_{i}=\boldsymbol{q}_{m}^{f} \cdot \boldsymbol{n}_{i} \quad \text { on } S_{i} .
$$

To comply with the macroscopic adiabatic assumption, the thermal boundary conditions of the fluid and the solid are given as

$$
\boldsymbol{q}_{m}^{s+} \cdot \boldsymbol{n}^{+}=-\boldsymbol{q}_{m}^{s^{-}} \cdot \boldsymbol{n}^{-} \text {on } S_{e}^{s} \quad \text { and } \quad \boldsymbol{q}_{m}^{f+} \cdot \boldsymbol{n}^{+}=-\boldsymbol{q}_{m}^{f-} \cdot \boldsymbol{n}^{-} \text {on } S_{e}^{f},
$$

so that the total thermal flux leaving the RVE is zero, i.e.

$$
\int_{S_{e}^{s}} \boldsymbol{q}_{m}^{S} \cdot \boldsymbol{n} \mathrm{d} A+\int_{S_{e}^{f}} \boldsymbol{q}_{m}^{f} \cdot \boldsymbol{n} \mathrm{d} A=0 .
$$

The next step is to obtain the expression of the microscopic energy variation. Satisfying energy conservation, the microscopic energy variation has to equal the sum of the variation of the external mechanical work and the variation of the heat:

$$
\int_{V} \delta E_{m} \mathrm{~d} V=\int_{\partial V^{s}} \boldsymbol{n} \cdot\left(\boldsymbol{\sigma}_{m}^{s} \cdot \delta \boldsymbol{u}_{m}^{s}-\frac{1}{\mathrm{j} \omega} \delta \boldsymbol{q}_{m}^{s}\right) \mathrm{d} A+\int_{\partial V^{f}} \boldsymbol{n} \cdot\left(\delta \boldsymbol{\sigma}_{m}^{f} \cdot \boldsymbol{u}_{m}^{f}-\frac{1}{\mathrm{j} \omega} \delta \boldsymbol{q}_{m}^{f}\right) \mathrm{d} A .
$$

Noticing the continuous interface conditions Eqs. (20) and (21), the integration boundaries reduce to

$$
\int_{V} \delta E_{m} \mathrm{~d} V=\int_{S_{e}^{s}} \boldsymbol{n} \cdot\left(\boldsymbol{\sigma}_{m}^{s} \cdot \delta \boldsymbol{u}_{m}^{s}-\frac{1}{\mathrm{j} \omega} \delta \boldsymbol{q}_{m}^{s}\right) \mathrm{d} A+\int_{S_{e}^{f}} \boldsymbol{n} \cdot\left(\delta \boldsymbol{\sigma}_{m}^{f} \cdot \boldsymbol{u}_{m}^{f}-\frac{1}{\mathrm{j} \omega} \delta \boldsymbol{q}_{m}^{f}\right) \mathrm{d} A .
$$

Substituting the boundary conditions Eqs. (18), (19) and (22) into this expression gives

$$
\int_{V} \delta E_{m} \mathrm{~d} V=\int_{S_{e}^{s}}\left(\boldsymbol{n} \cdot \boldsymbol{\sigma}_{m}^{s}\right) \mathrm{d} A \cdot \delta \boldsymbol{u}_{M}^{s}+\int_{S_{e}^{s}}\left(\boldsymbol{n} \cdot \boldsymbol{\sigma}_{m}^{s}\right) \boldsymbol{x}_{m}^{s} \mathrm{~d} A: \delta\left(\nabla_{M} \boldsymbol{u}_{M}^{s}\right)+\int_{S_{e}^{s}} \boldsymbol{n} \cdot \boldsymbol{\sigma}_{m}^{s} \cdot \delta \tilde{\boldsymbol{u}}_{m}^{s} \mathrm{~d} A-\delta p_{M}^{f} \int_{S_{e}^{f}} \boldsymbol{n} \cdot \boldsymbol{u}_{m}^{f} \mathrm{~d} A-\delta \nabla_{M} p_{M}^{f} \cdot \int_{S_{e}^{f}} \boldsymbol{x}_{m}^{f}\left(\boldsymbol{n} \cdot \boldsymbol{u}_{m}^{f}\right) \mathrm{d} A .
$$

The integration involving the solid displacement fluctuation is zero because of the periodic boundary condition and the constraints on the corner points. Therefore, the microscopic energy variation can be written as

$$
\int_{V} \delta E_{m} \mathrm{~d} V=\int_{S_{e}^{s}}\left(\boldsymbol{n} \cdot \boldsymbol{\sigma}_{m}^{s}\right) \mathrm{d} A \cdot \delta \boldsymbol{u}_{M}^{s}+\int_{S_{e}^{s}}\left(\boldsymbol{n} \cdot \boldsymbol{\sigma}_{m}^{s}\right) \boldsymbol{x}_{m}^{S} \mathrm{~d} A: \delta\left(\nabla_{M} \boldsymbol{u}_{M}^{s}\right)-\delta p_{M}^{f} \int_{S_{e}^{f}} \boldsymbol{n} \cdot \boldsymbol{u}_{m}^{f} \mathrm{~d} A-\delta \nabla_{M} p_{M}^{f} \cdot \int_{S_{e}} \boldsymbol{x}_{m}^{f}\left(\boldsymbol{n} \cdot \boldsymbol{u}_{m}^{f}\right) \mathrm{d} A .
$$




\subsection{Micro-to-macro relations}

Energy consistency requires that the energy variation of a macroscopic point per unit of volume is the volume average of the total microscopic energy variation of the associated RVE, i.e.

$$
\delta E_{M}=\frac{1}{V} \int_{V} \delta E_{m} \mathrm{~d} V
$$

Substituting Eqs. (5) and (27) into this equation results in the following micro-to-macro relations:

$$
\begin{aligned}
(1-\phi) \boldsymbol{f}_{M}^{S} & =\frac{1}{V} \int_{S_{e}^{s}}\left(\boldsymbol{n} \cdot \boldsymbol{\sigma}_{m}^{S}\right) \mathrm{d} A, \quad(1-\phi) \boldsymbol{\sigma}_{M}^{S}=\frac{1}{V} \int_{S_{e}^{s}}\left(\boldsymbol{n} \cdot \boldsymbol{\sigma}_{m}^{S}\right) \boldsymbol{x}_{m} \mathrm{~d} A \\
\phi \epsilon_{M}^{f} & =\frac{1}{V} \int_{S_{e}^{f}} \boldsymbol{n} \cdot \boldsymbol{u}_{m}^{f} \mathrm{~d} A, \quad \phi \boldsymbol{u}_{M}^{f}=\frac{1}{V} \int_{S_{e}^{f}}\left(\boldsymbol{n} \cdot \boldsymbol{u}_{m}^{f}\right) \boldsymbol{x}_{m}^{f} \mathrm{~d} A .
\end{aligned}
$$

Because of the periodic thermal flux condition, Eq. (22), the expressions in Eq. (29) are identical to those for isothermal conditions [49]. However, the microscopic temperature field still affects the microscopic displacement fields.

Considering the linearity of the microscopic problem, the macroscopic quantities calculated from Eq. (29) can be linearly expressed in terms of the macroscopic solid displacement, air pressure and their gradients. As shown in previous work [49], the effects of $\left(\nabla_{M} \boldsymbol{u}_{M}^{s}, p_{M}^{f}\right)$ on $\left((1-\phi) \boldsymbol{f}_{M}^{s}, \phi \boldsymbol{u}_{M}^{f}\right)$ depend on the scale ratio $R / L_{M}^{*}$. When the scale separation principle of Eq. (2) is satisfied, the solid displacement $\boldsymbol{u}_{M}^{s}$ and the pressure gradient $\nabla_{M} p_{M}^{f}$ are the dominant factors for the response of the RVE:

$$
\left[\begin{array}{c}
(1-\phi) \boldsymbol{f}_{M}^{s} \\
\phi \boldsymbol{u}_{M}^{f}
\end{array}\right]=\left[\begin{array}{cc}
-\omega^{2} \rho_{0}^{s} \boldsymbol{K}^{s} & \boldsymbol{K}^{s f} \\
\left(\boldsymbol{K}^{s f}\right)^{\mathrm{T}} & \left(\omega^{2} \rho_{0}^{f}\right)^{-1} \boldsymbol{K}^{f}
\end{array}\right] \cdot\left[\begin{array}{c}
\boldsymbol{u}_{M}^{s} \\
\nabla_{M} p_{M}^{f}
\end{array}\right],
$$

where the dimensionless coefficients $\boldsymbol{K}^{s}, \boldsymbol{K}^{f}$ and $\boldsymbol{K}^{s f}$ are 2nd-order tensors. Here, the diagonal terms should be the transpose of each other because Eq. (5) gives

$$
\frac{(1-\phi) \delta \boldsymbol{f}_{M}^{s}}{\delta \nabla_{M} p_{M}^{f}}=\frac{\delta^{2} E_{M}}{\delta \nabla_{M} p_{M}^{f} \delta \boldsymbol{u}_{M}^{s}}=\left(\frac{\delta^{2} E_{M}}{\delta \boldsymbol{u}_{M}^{s} \delta \nabla_{M} p_{M}^{f}}\right)^{\mathrm{T}}=\left(\frac{\phi \delta \boldsymbol{u}_{M}^{f}}{\delta \boldsymbol{u}_{M}^{s}}\right)^{\mathrm{T}},
$$

where the variation of a vector $\boldsymbol{y}$ with respect to a vector $\boldsymbol{x}$ and the second-order variation of a scalar $E$ with respect to two vectors $\boldsymbol{a}$ and $\boldsymbol{b}$ are written as

$$
\left(\frac{\delta \boldsymbol{y}}{\delta \boldsymbol{x}}\right)_{i j}=\frac{\delta y_{i}}{\delta x_{j}} \text { and }\left(\frac{\delta^{2} E}{\delta \boldsymbol{a} \delta \boldsymbol{b}}\right)_{i j}=\frac{\delta^{2} E}{\delta a_{j} \delta b_{i}}
$$

In this paper, for simplicity, it is assumed that the motion in $i$ direction does not influence the motion in $j$ direction (for $i \neq j$ ). Then, the three tensors $\boldsymbol{K}^{s}, \boldsymbol{K}^{f}$ and $\boldsymbol{K}^{s f}$ are all diagonal. Similarly, the solid stress $\boldsymbol{\sigma}_{M}^{s}$ and the fluid volumetric change $\epsilon_{M}^{f}$ are mainly affected by the solid deformation $\nabla_{M} \boldsymbol{u}_{M}^{s}$ and the fluid pressure $p_{M}^{f}$ [49]:

$$
\begin{gathered}
(1-\phi) \boldsymbol{\sigma}_{M}^{s}={ }^{4} \boldsymbol{C}^{s}: \nabla_{M} \boldsymbol{u}_{M}^{s}-\boldsymbol{\Psi}^{s f} p_{M}^{f} \\
\phi \epsilon_{M}^{f}=-\boldsymbol{\Psi}^{s f}: \nabla_{M} \boldsymbol{u}_{M}^{s}-S^{f} p_{M}^{f},
\end{gathered}
$$

where the 4th-order tensor ${ }^{4} \boldsymbol{C}^{S}$ is the stiffness tensor of the solid skeleton and the scalar $S^{f}$ is the compliance of the air. The coupling term is a 2nd-order tensor $\Psi^{s f}$. In fact, substituting Eqs. (30) and (33) into Eq. (3) results in a closed set of differential equations at the macroscopic scale with respect to $\boldsymbol{u}_{M}^{s}$ and $p_{M}^{f}$. The closed macroscopic problem requires the effective parameters $\left(\boldsymbol{K}^{s}, \boldsymbol{K}^{f}, \boldsymbol{K}^{s f}\right)$ and $\left({ }^{4} \boldsymbol{C}^{s}, \boldsymbol{\Psi}^{s f}, S^{f}\right)$ that are extracted from the associated microscopic problem.

\subsection{Relation with Biot's poroelastic theory}

In this part, the relation between the homogenization result and Biot's poroelastic theory is discussed. Firstly, by moving $\boldsymbol{u}_{M}^{f}$ to the right hand side and moving $\nabla_{M} p_{M}^{f}$ to the left hand side, Eq. (30) can be rewritten as

$$
\begin{gathered}
(1-\phi) \boldsymbol{f}_{M}^{s}=-\omega^{2} \rho_{0}^{s} \boldsymbol{\eta}^{s} \cdot \boldsymbol{u}_{M}^{s}+\omega^{2} \boldsymbol{\rho}_{c} \cdot\left(\boldsymbol{u}_{M}^{f}-\boldsymbol{u}_{M}^{s}\right) \\
-\phi \nabla_{M} p_{M}^{f}=-\omega^{2} \boldsymbol{\rho}_{c} \cdot\left(\boldsymbol{u}_{M}^{f}-\boldsymbol{u}_{M}^{s}\right)-\omega^{2} \rho_{0}^{f} \boldsymbol{\eta}^{f} \cdot \boldsymbol{u}_{M}^{f},
\end{gathered}
$$

with

$$
\boldsymbol{\eta}^{s}=\boldsymbol{K}^{s}-\frac{\rho_{0}^{f}}{\rho_{0}^{s}} \boldsymbol{K}^{s f} \cdot\left(\boldsymbol{K}^{f}\right)^{-1} \cdot\left(\phi \mathbf{I}-\boldsymbol{K}^{s f}\right), \quad \boldsymbol{\eta}^{f}=\left(\phi^{2} \mathbf{I}-\phi \boldsymbol{K}^{s f}\right) \cdot\left(\boldsymbol{K}^{f}\right)^{-1}, \quad \boldsymbol{\rho}_{c}=\phi \rho_{0}^{f} \boldsymbol{K}^{s f} \cdot\left(\boldsymbol{K}^{f}\right)^{-1} .
$$

Since $\boldsymbol{K}^{s}, \boldsymbol{K}^{f}$ and $\boldsymbol{K}^{s f}$ are all diagonal, $\left(\boldsymbol{\eta}^{s}, \boldsymbol{\eta}^{f}, \boldsymbol{\rho}_{c}\right)$ are diagonal 2nd-order tensors as well. Summing up the two equations gives

$$
(1-\phi) \boldsymbol{f}_{M}^{s}-\phi \nabla_{M} p_{M}^{f}=-\omega^{2} \rho_{0}^{s} \eta^{s} \cdot \boldsymbol{u}_{M}^{s}-\omega^{2} \rho_{0}^{f} \boldsymbol{\eta}^{f} \cdot \boldsymbol{u}_{M}^{f}
$$


It can also be shown that [49]

$$
\begin{aligned}
(1-\phi) \boldsymbol{f}_{M}^{s}-\phi \nabla_{M} p_{M}^{f}= & -\omega^{2} \rho_{0}^{s}\left(\frac{1}{V} \int_{V^{s}} \boldsymbol{u}_{m}^{s} \mathrm{~d} V\right)-\omega^{2} \rho_{0}^{f}\left(\frac{1}{V} \int_{V^{f}} \boldsymbol{u}_{m}^{f} \mathrm{~d} V\right)=-\omega^{2} \rho_{0}^{s}\left[(1-\phi) \boldsymbol{u}_{M}^{s}+\frac{1}{V} \int_{V^{s}} \tilde{\boldsymbol{u}}_{m}^{s} \mathrm{~d} V\right] \\
& -\omega^{2} \rho_{0}^{f}\left[\phi \boldsymbol{u}_{M}^{f}-\frac{1}{V} \int_{V^{f}}\left(\nabla_{m} \cdot \boldsymbol{u}_{m}^{f}\right) \boldsymbol{x}_{m}^{f} \mathrm{~d} V+\frac{1}{V} \int_{S_{i}} \boldsymbol{x}_{m}^{f}\left(\boldsymbol{n}_{i} \cdot \boldsymbol{u}_{m}^{f}\right) \mathrm{d} A\right] .
\end{aligned}
$$

Comparing the above two equations gives

$$
\begin{gathered}
\boldsymbol{\eta}^{s} \cdot \boldsymbol{u}_{M}^{s}=(1-\phi) \boldsymbol{u}_{M}^{s}+\frac{1}{V} \int_{V^{s}} \tilde{\boldsymbol{u}}_{m}^{s} \mathrm{~d} V \\
\boldsymbol{\eta}^{f} \cdot \boldsymbol{u}_{M}^{f}=\phi \boldsymbol{u}_{M}^{f}-\frac{1}{V} \int_{V^{f}}\left(\nabla_{m} \cdot \boldsymbol{u}_{m}^{f}\right) \boldsymbol{x}_{m}^{f} \mathrm{~d} V+\frac{1}{V} \int_{S_{i}} \boldsymbol{x}_{m}^{f}\left(\boldsymbol{n}_{i} \cdot \boldsymbol{u}_{m}^{f}\right) \mathrm{d} A .
\end{gathered}
$$

When the microscopic fluctuation $\tilde{\boldsymbol{u}}_{m}^{s}$ can be ignored and the volume-average fluid deformation is small enough, it is reasonable to apply the following approximation:

$$
\boldsymbol{\eta}^{s} \approx(1-\phi) \mathbf{I} \text { and } \boldsymbol{\eta}^{f} \approx \phi \mathbf{I}
$$

Then, the static densities are used in the right hand side of Eq. (36). Note that Eq. (39) is only valid when the microscopic fluctuation can be ignored and the average deformation remains small. When these two conditions are not satisfied, the dynamic terms, i.e. $\boldsymbol{\eta}^{s}$ and $\boldsymbol{\eta}^{f}$, should be used. In the following discussion on the numerical results, it will be shown that the microscopic fluctuation can be significant in some cases. By using Eq. (39), Eq. (34) leads to the anisotropic Biot's poroelastic equations [26]:

$$
\begin{gathered}
(1-\phi) \boldsymbol{f}_{M}^{s}-\phi \nabla_{M} p_{M}^{f}=-\omega^{2}(1-\phi) \rho_{0}^{s} \boldsymbol{u}_{M}^{s}-\omega^{2} \phi \rho_{0}^{f} \boldsymbol{u}_{M}^{f} \\
-\phi \nabla_{M} p_{M}^{f}+\omega^{2} \phi \rho_{0}^{f} \boldsymbol{u}_{M}^{f}=-\omega^{2} \boldsymbol{\rho}_{c} \cdot\left(\boldsymbol{u}_{M}^{f}-\boldsymbol{u}_{M}^{s}\right) .
\end{gathered}
$$

The term $\mathrm{j} \omega \boldsymbol{\rho}_{c} / \phi$ has the same physical meaning as the viscodynamic operator defined in [26]. Combining Eqs. (39) and (35) gives the expression of this viscodynamic operator

$$
\frac{\mathrm{j} \omega \boldsymbol{\rho}_{c}}{\phi}=\mathrm{j} \omega \rho_{0}^{f}\left(\phi \mathbf{I}-\boldsymbol{K}^{f}\right) \cdot\left(\boldsymbol{K}^{f}\right)^{-1}
$$

where $\boldsymbol{K}^{f}$ is determined from the microscopic RVE.

For the stress-strain relation, rewriting Eq. (33) gives

$$
\begin{aligned}
(1-\phi) \boldsymbol{\sigma}_{M}^{s} & ={ }^{4} \boldsymbol{D}: \nabla_{M} \boldsymbol{u}_{M}^{s}+\boldsymbol{Q} \epsilon_{M}^{f} \\
-\phi p_{M}^{f} & =\boldsymbol{Q}: \nabla_{M} \boldsymbol{u}_{M}^{s}+R \epsilon_{M}^{f},
\end{aligned}
$$

with

$$
{ }^{4} \boldsymbol{D}={ }^{4} \boldsymbol{C}^{s}+\frac{1}{S^{f}} \boldsymbol{\Psi}^{s f} \boldsymbol{\Psi}^{s f}, \quad \boldsymbol{Q}=\frac{\phi}{S^{f}} \boldsymbol{\Psi}^{s f}, \quad R=\frac{\phi^{2}}{S^{f}} .
$$

This is identical to the stress-strain relation in Biot's theory [25]. Instead of the macroscopic measurements required in Biot's theory, the effective material parameters $\left({ }^{4} \boldsymbol{D}, \boldsymbol{Q}, R\right)$ can now be numerically calculated based on the associated microscopic RVE.

To summarize, in the currently proposed homogenization approach, the effective tensorial parameters $\left(\boldsymbol{\eta}^{s}, \boldsymbol{\eta}^{f}, \boldsymbol{\rho}_{c}\right)$ and $\left({ }^{4} \boldsymbol{D}, \boldsymbol{Q}, R\right)$ are numerically calculated from the microscopic RVE and subsequently used in the closed-form macroscopic equations i.e. Eqs. (3), (34) and (42). In the particular case when the solid displacement fluctuation can be ignored and the volume average of the fluid deformation is small, the macroscopic governing equations reduce to Biot's poroelastic theory,

(a)

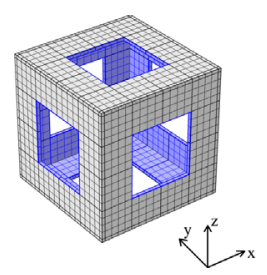

Solid domain (1352 elements) (b)

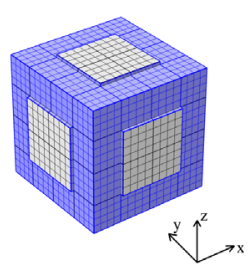

Fluid domain (4480 elements)

Fig. 2. Finite element model of a $1-\mathrm{mm}$ cubic RVE with a porosity of 0.895 percent. The membrane thickness is $50 \mu \mathrm{m}$, i.e. the wall thickness in (a) is $25 \mu \mathrm{m}$. The size of the face holes in the RVE is $0.5 \times 0.5 \mathrm{~mm}^{2}$. The parts of the boundaries of both domains indicated with a blue color represent the fluid-solid interfaces. (a) Solid domain (1352 elements). (b) Fluid domain (4480 elements). (For interpretation of the references to color in this figure caption, the reader is referred to the web version of this paper.) 
Table 1

Material parameters of aluminum ( $\mathrm{Al}$ ) and two polyurethanes (PU) used in the simulations. The mechanical properties of PU are estimated from [51] and the thermal properties are estimated from [52].

\begin{tabular}{|c|c|c|c|c|c|}
\hline Material & $\rho_{0}^{s}\left(\mathrm{~kg} \mathrm{~m}^{-3}\right)$ & $K^{S}(\mathrm{GPa})$ & $G^{s}(\mathrm{GPa})$ & $C_{p}^{S}\left(\mathrm{~J} \mathrm{~kg}^{-1} \mathrm{~K}^{-1}\right)$ & $k^{S}\left(\mathrm{~W} \mathrm{~m}{ }^{-1} \mathrm{~K}^{-1}\right)$ \\
\hline $\mathrm{Al}$ & 2700 & 76 & 26 & 910 & 205 \\
\hline PU A & 1100 & 2.48 & 0.33 & 1800 & 0.022 \\
\hline PU B & 1100 & 0.22 & 0.083 & 1800 & 0.022 \\
\hline
\end{tabular}

Table 2

Loading conditions used to calculate $\left(\eta^{s}, \eta^{f}, \rho_{c}\right)$. Here $\boldsymbol{e}_{x}$ is the unit vector in $x$ direction. The solid deformation $\nabla_{M} \boldsymbol{u}_{M}^{s}$ and the fluid pressure $p_{M}^{f}$ are both zero.

\begin{tabular}{ll}
\hline Set & $\boldsymbol{u}_{M}^{s}(\mathrm{~m})$ \\
\hline 1 & $\nabla_{M}^{f} p_{M}(\mathrm{~Pa} / \mathrm{m})$ \\
2 & 0 \\
$10^{-4} \boldsymbol{e}_{x}$ & 0 \\
$10^{4} \boldsymbol{e}_{x}$
\end{tabular}

where the effective material parameters $\left({ }^{4} \boldsymbol{D}, \boldsymbol{Q}, R\right)$ and the viscodynamic operator $\mathrm{j} \omega \boldsymbol{\rho}_{c} / \phi$ are numerically calculated. The full set of closed-form macroscopic equations with parameters obtained from the microscopic RVE is referred to as the GeneralBalance-Law-Computational-Homogenization (GBL-CH) model, whereas Biot's poroelastic model with parameters obtained from the microscopic RVE is referred to as the Biot-Computational-Homogenization (Biot-CH) model. Clearly, the GBL-CH model is expected to be more versatile for cases with significant microscopic fluctuations than the Biot-CH model.

\section{Microscopic simulation results}

In the previous section, two models have been presented: the GBL-CH model and the Biot-CH model. In this section, the numerical results of a cubic RVE with idealized partially open membranes as shown in Fig. 2 are discussed. Attention is focused on the influences of the non-isothermal condition and the microscopic fluctuation of the solid displacement. The effective parameters are investigated: the first part focuses on the coefficients $\left(\boldsymbol{\eta}^{s}, \boldsymbol{\eta}^{f}, \boldsymbol{\rho}_{c}\right)$, whereas $(\boldsymbol{Q}, R)$ are studied in the second part. The fluid considered is air whereas three different solid materials are used as shown in Table 1: aluminum (Al) and two polyurethanes (PU) with a different stiffness. The effective parameters of the two models obtained with the homogenization method are compared with the results of the Biot-JCAL model, where the JCAL model is adopted to determine Biot's parameters. All simulations are conducted in COMSOL 4.3b by using quadratic elements in the weak form PDE module.

In the GBL-CH model, the coefficients $\left(\boldsymbol{\eta}^{s}, \boldsymbol{\eta}^{f}, \boldsymbol{\rho}_{c}\right)$ are calculated through Eq. (35) with $\left(\boldsymbol{K}^{s}, \boldsymbol{K}^{f}, \boldsymbol{K}^{s f}\right)$ obtained inversely from Eq. (30). The inverse calculation of $\left(\boldsymbol{K}^{s}, \boldsymbol{K}^{f}, \boldsymbol{K}^{s f}\right)$ is based on six simulations of the microscopic RVE, where Eqs. (18), (19) and (22) are adopted by applying $\nabla_{M} \boldsymbol{u}_{M}^{s}=\mathbf{0}, p_{M}^{f}=0$ and six different $\left(\boldsymbol{u}_{M}^{s}, \nabla_{M} p_{M}^{f}\right)$. Considering the cubic symmetry, all tensor components can be represented in terms of a set of scalars such as $\left(\eta^{s}, \eta^{f}, \rho_{c}\right)$ and only two simulations are necessary for the cubic RVE in Fig. 2. The specific values of non-zero components of $\left(\boldsymbol{u}_{M}^{s}, \nabla_{M} p_{M}^{f}\right)$ in $x$ direction used for the calculation in this paper can be found in Table 2 .

According to Eq. (34), the effective fluid density is expressed as $\rho_{\text {eff }}^{f}=\rho_{0}^{f} \eta^{f}+\rho_{c}$ by letting $\boldsymbol{u}_{M}^{s}=\mathbf{0}$. Since the viscodynamic operator in the Biot-CH model is obtained by Eq. (41), combined with Eq. (39), the effective fluid density of the Biot-CH model is the same as the one of the GBL-CH model. In the Biot-JCAL model, the viscodynamic operator is expressed as [2]

$$
\phi \rho_{0}^{f}+\rho_{c}=\phi \rho_{0}^{f} \alpha_{\infty}+\frac{\phi^{2} \mu^{f}}{\mathrm{j} \omega q_{0}}\left[1+\frac{\mathrm{j} \omega \rho_{0}^{f}}{\mu^{f}}\left(\frac{2 \alpha_{\infty} q_{0}}{\Lambda \phi}\right)^{2}\right]^{1 / 2} .
$$

The required non-acoustical parameters are numerically calculated based on the RVE according to the method in [11]: the tortuosity at infinite frequency $\alpha_{\infty}$ is 1.58 , the static permeability $q_{0}$ is $6.43 \times 10^{-9} \mathrm{~m}^{2}$ and the viscous characteristic length $\Lambda$ is $3.29 \times 10^{-4} \mathrm{~m}$.

For the different solid properties considered, the maximum relative difference of the effective fluid densities is still smaller than 0.01 percent in the GBL-CH model. Therefore, the result of $\mathrm{Al}$ is plotted in Fig. 3 to compare with the isothermal results from previous work [49]. In this figure, the curves of the Biot-CH model and the GBL-CH model are exactly overlapping. Compared with the isothermal results (the gray curves), the non-isothermal results have smaller magnitudes. Hence, the non-isothermal case will have a higher fluid velocity than the isothermal case for the same applied pressure gradient. Besides, the magnitude in the GBL-CH model is larger than in the Biot-JCAL model, while these two models have almost the same result at high frequencies. It also shows that the GBL-CH model predicts a slightly smaller phase around $100-200 \mathrm{~Hz}$ but a significantly larger phase after $500 \mathrm{~Hz}$. According to Biot's theory, the phase of the viscodynamic operator 
(a)

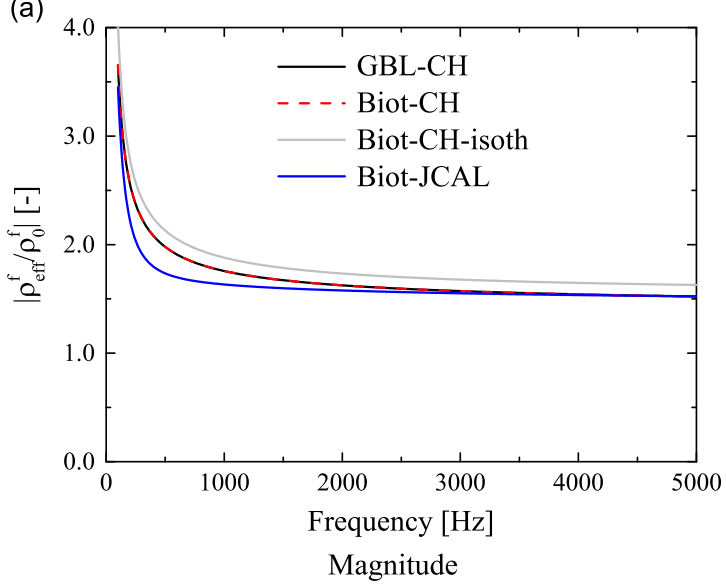

(b)

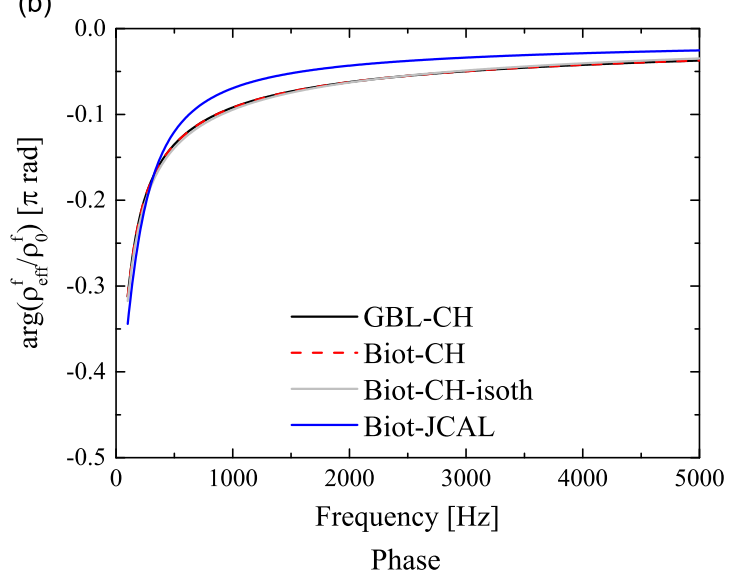

Fig. 3. Normalized effective fluid densities of various models with $\mathrm{Al}$ as the solid. (a) Magnitude. (b) Phase.

(a)

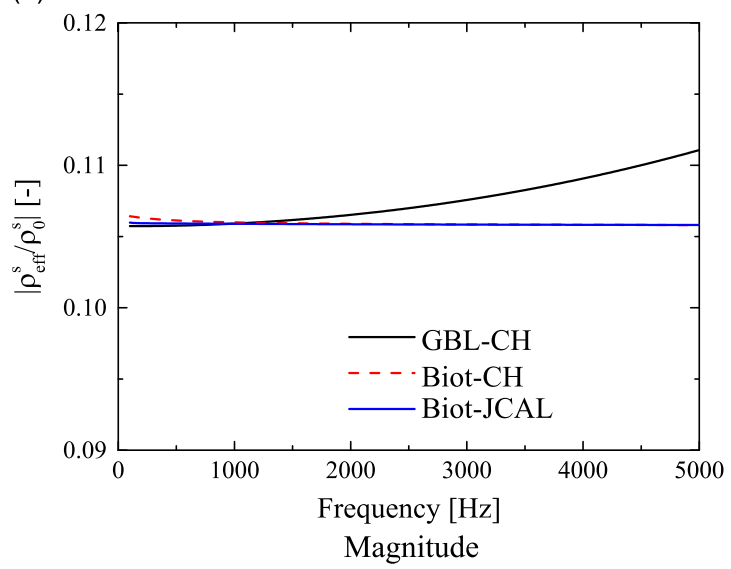

(b)

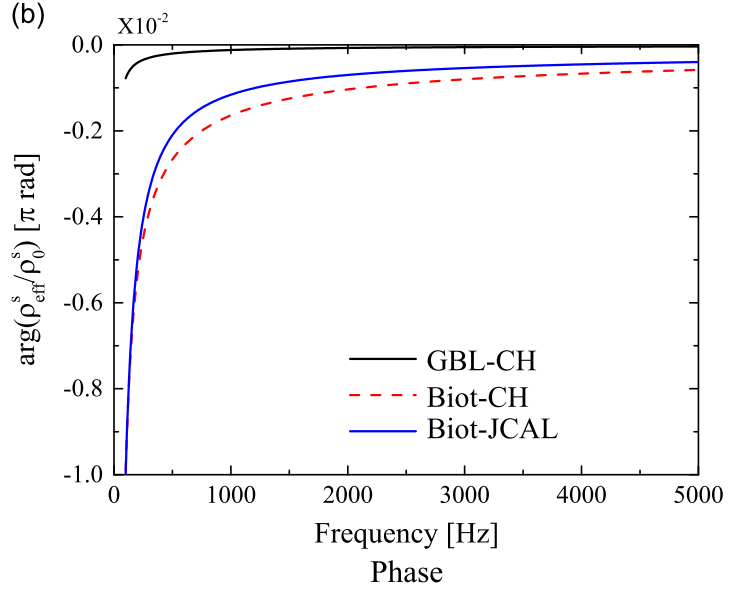

Fig. 4. Normalized effective solid densities of various models for PU B. (a) Magnitude. (b) Phase.

represents the relaxation time associated with dissipative processes in porous materials [26] and a longer relaxation time means more dissipation, leading to a better absorption performance, which will be shown in the next section.

Similarly, the effective solid density can be calculated as $\rho_{\text {eff }}^{s}=\rho_{0}^{s} \eta^{s}+\rho_{c}$. When normalized by the static solid density, it is close to $1-\phi$ in both the Biot-JCAL model and the Biot- $\mathrm{CH}$ model because of the significant difference between the solid density and the air density. Fig. 4(a) reveals that the magnitudes of the densities obtained by the Biot-JCAL and the Biot- $\mathrm{CH}$ models are very close to 0.105 . It decreases with increasing frequency, but the decrease is smaller than 0.5 percent from 100 to $5000 \mathrm{~Hz}$. However, the GBL-CH model gives a different behavior: the density increases significantly (almost 5 percent from 100 to $5000 \mathrm{~Hz}$ ) when the frequency increases. Since the difference between the two homogenization models is the ignorance of the microscopic solid fluctuations, it suggests that the microscopic fluctuations may be significant for materials with a low stiffness at high frequencies. On the other hand, because the solid in the microscopic RVE is purely elastic, the phase of the solid density reflects the relaxation time of the thermal dissipation in the solid and the time lag between the fluid displacement and the solid displacement. Fig. 4(b) shows that the phase in the result of the GBL-CH model is much smaller than for the other two models, suggesting that the heat and the momentum transfers between the fluid and the solid described by the GBL-CH model are much faster than the other models. However, note that all three phases are small and the effect on the difference between the phases is not as significant as on the magnitude.

Now, the effects of the solid stiffness on the effective solid density are studied. In the Biot-JCAL model, the normalized effective solid density is expressed as $(1-\phi)+\rho_{c} / \rho_{0}^{s}$ and it is not influenced by the solid stiffness. Because the solid stiffness has only a small influence on $\boldsymbol{\rho}_{c}$ (as shown in the previous discussion on the effective fluid density), the influence of the solid stiffness is negligible in the Biot-CH model. However, the GBL-CH model gives a different result: the solid stiffness may affect the effective solid density through the microscopic fluctuations. When the solid changes from Al to PU A, the magnitude of the effective solid density increases slightly as shown in Fig. 5(a) (around 0.3 percent). Although the relative difference between PU A and PU B is smaller than 0.01 percent at low frequencies, it increases to 5 percent at $5000 \mathrm{~Hz}$. It shows that the solid stiffness effectively alters the magnitude of the effective solid density. Moreover, because PU A and PU B have 
(a)

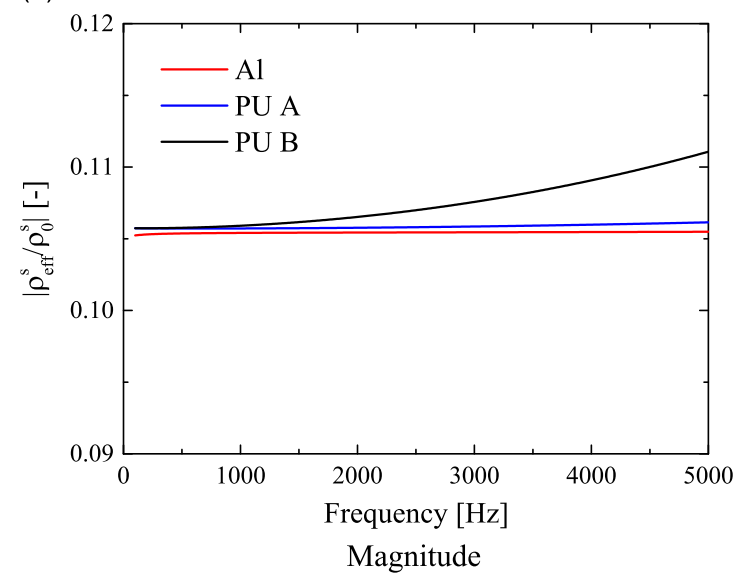

(b)

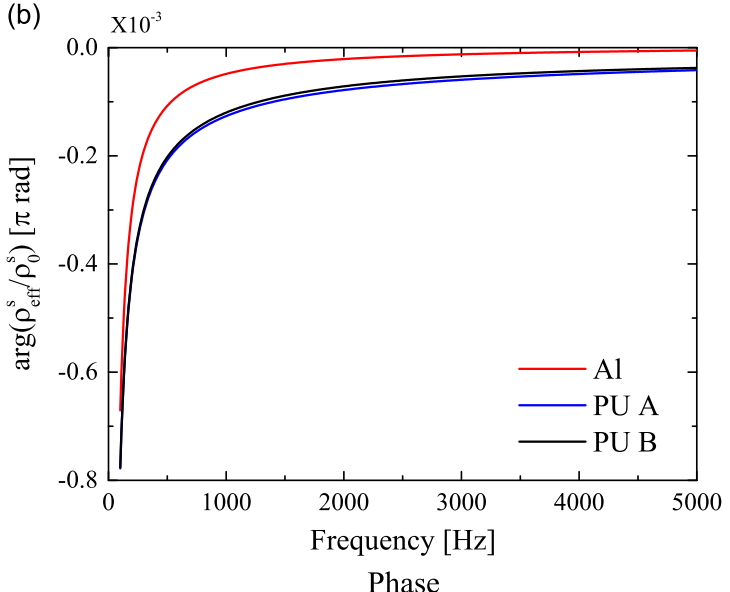

Fig. 5. Comparison of effective solid densities of the GBL-CH model for various solid materials. (a) Magnitude. (b) Phase.

Table 3

Loading conditions used to calculate $\left({ }^{4} \boldsymbol{D}, Q, R\right)$. Here $\boldsymbol{e}_{x}$ and $\boldsymbol{e}_{y}$ are the unit vectors in $x$ and $y$ directions, respectively. The solid displacement $\boldsymbol{u}_{M}^{s}$ and the fluid pressure gradient $\nabla_{M} p_{M}^{f}$ are both zero.

\begin{tabular}{lll}
\hline Set & $\nabla_{M} \boldsymbol{u}_{M}^{s}(-)$ & $p_{M}^{f}(\mathrm{~Pa})$ \\
\hline 1 & $10^{-4} \boldsymbol{e}_{x} \boldsymbol{e}_{x}$ & 1 \\
2 & $10^{-4} \boldsymbol{e}_{y} \boldsymbol{e}_{y}$ & 1 \\
3 & $10^{-4} \boldsymbol{e}_{x} \boldsymbol{e}_{y}$ & 1 \\
4 & $\mathbf{0}$ & 1 \\
\hline
\end{tabular}

almost the same phases in Fig. 5(b), it means that the solid stiffness has very small influences on the relaxation time of the solid.

The effective material parameters $\left({ }^{4} \boldsymbol{D}, \boldsymbol{Q}, R\right)$ are calculated through Eq. (43) with the coefficients $\left({ }^{4} \boldsymbol{C}^{S}, \boldsymbol{\Psi}^{s f}, S^{f}\right)$ obtained from the reverse calculation of Eq. (33). Analogously, the reverse calculation requires ten simulations with different loading conditions of $\left(\nabla_{M} \boldsymbol{u}_{M}^{s}, p_{M}^{f}\right)$. For the cubic RVE, there are only three independent parameters in ${ }^{4} \boldsymbol{C}^{s}$. Since the origin is set in the geometrical center, the shear deformation of the solid skeleton does not influence the volumetric change of the fluid. Considering the cubic symmetry, $\Psi=\Psi \mathbf{I}$, making $\boldsymbol{Q}=Q \mathbf{I}$ with the scalar $Q=\phi \Psi / S^{f}$. Hence, only four simulations involving at least one shear deformation, one expansion and one non-zero pressure loading are required to obtain $\left({ }^{4} \boldsymbol{D}, Q, R\right)$. The specific values used for this are given in Table 3. Because both the Biot-CH model and the GBL-CH model use Eqs. (42) and (43) in the stress-strain relation, only the result of the GBL-CH model is presented.

According to Eq. (42), $(Q, R)$ are directly related to the fluid pressure. The relative difference between $R$ for $A l$ and $R$ for PU A is around 0.7 percent at $100 \mathrm{~Hz}$ and gradually decreases to around 0.2 percent at $5000 \mathrm{~Hz}$. The corresponding $R$ for PU A and for PU B are the same. The maximum relative difference of $Q$ for $\mathrm{Al}$ and for PU A (at $100 \mathrm{~Hz}$ ) is around 0.7 percent and the difference between the values for PU A and PU B is smaller than 0.01 percent (at $100 \mathrm{~Hz}$ ). The difference between Al and PU A being more significant than the difference between PU A and PU B suggests that the thermal properties of the solid are much more important for $(Q, R)$ than the solid stiffness and the influence of the thermal properties decreases with increasing frequency.

First, the homogenization results are compared with the isothermal result. As the differences of $(Q, R)$ between various solid materials are small, the result for $\mathrm{Al}$ is compared with the isothermal result in Fig. 6. As shown by the gray curves representing the isothermal result, $R$ and $Q$ are real-valued and almost constant at $0.90 P_{0}$ and $0.73 P_{0}$, respectively. In the non-isothermal result (the black curves), the two parameters become complex and frequency-dependent functions whose magnitudes are increasing with increasing frequency. The key change for the non-isothermal condition is the imaginary part of the effective material parameters $(R, Q)$, where the thermal dissipation is modifying in particular the low frequency behavior.

Second, the difference between the GBL-CH model and the Biot-JCAL model is checked. In the Biot-JCAL model, ( $Q, R)$ are expressed as:

$$
R=\frac{\phi^{2} K_{\mathrm{eff}}^{f}}{\phi+\left(K_{\mathrm{eff}}^{f} / K^{s}\right)\left(1-\phi-K_{b} / K^{s}\right)} \quad \text { and } \quad Q=\left(\phi-\frac{R}{K_{\mathrm{eff}}^{f}}\right) K^{s}
$$



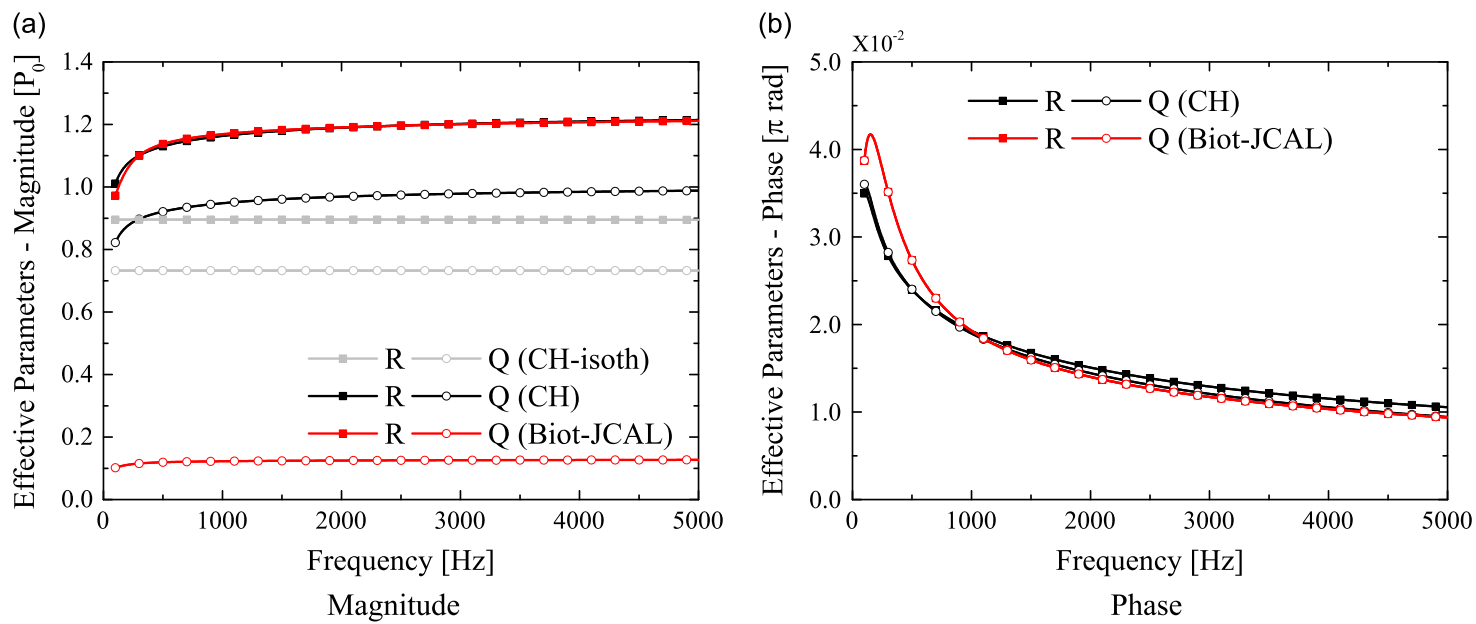

Fig. 6. Comparison of $R$ and $Q$ obtained from the Biot-JCAL model and the GBL-CH model for Al. (a) Magnitude. (b) Phase.

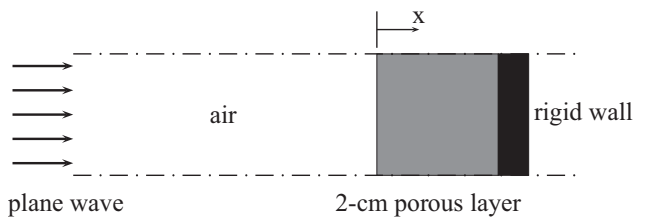

Fig. 7. Macroscopic configuration for obtaining normal incident sound absorption coefficients. Symmetry boundary conditions are applied at the top and bottom.

Here $K_{b}$ is the effective bulk modulus of the solid skeleton which is obtained according to Eq. (5-3) in [53]. The effective bulk modulus of the fluid $K_{\text {eff }}^{f}$ is calculated according to [3,4]

$$
K_{\mathrm{eff}}^{f}=P_{0}\left[1-\left(1-\frac{1}{\gamma}\right)\left(1+\frac{\phi \mu_{f}}{\mathrm{j} \omega q_{0}^{\prime} \rho_{0}^{f} \operatorname{Pr}} \sqrt{1+\frac{\mathrm{j} \omega \rho_{0}^{f} \operatorname{Pr}}{\mu_{f}}\left(\frac{2 q_{0}^{\prime}}{\phi \Lambda^{\prime}}\right)^{2}}\right)^{-1}\right]^{-1},
$$

where $\gamma$ is the heat capacity ratio, $\operatorname{Pr}$ is the Prandtl number, the required thermal characteristic length $\Lambda^{\prime}$ is $4.25 \times 10^{-4} \mathrm{~m}$ and the static thermal permeability $q_{0}^{\prime}$ is $1.95 \times 10^{-8} \mathrm{~m}^{2}$, both calculated based on [11]. As shown in Fig. 6, the two models give almost the same behaviors for $R$ especially in terms of the magnitude. For the phase, similar as for the effective fluid densities in Fig. 3(b), the GBL-CH model predicts a shorter relaxation time at low frequencies but a longer time at high frequencies. However, the magnitudes of $Q$ are quite different: the GBL-CH model provides a considerably larger result than the Biot-JCAL model. Because the ratio $K_{\text {eff }}^{f} / K^{S}$ is very small, $R$ is mainly determined by $K_{\text {eff }}^{f}$ in Eq. (45). Nevertheless, $Q$ is affected by both $K_{\text {eff }}^{f}$ and $K_{b}$. The small differences in $R$ in Fig. 6 suggest that $K_{\text {eff }}^{f}$ described by the homogenization model and the Biot-JCAL model are very close. The large difference in $Q$ shows that $K_{b}$ obtained from the homogenization model is considerably different from the empirical relation in Eq. (5-3) in [53]. Noting that the empirical relation is used for open-cell foams, this large difference shows that the thin walls have significant influences on the mechanical properties.

As discussed above, the thermal effect is included through the incorporation of non-isothermal conditions. By including the microscopic fluctuation field in the solid displacement, the GBL-CH model gives a different description of the effective solid density compared with the Biot- $\mathrm{CH}$ model and the Biot-JCAL model. At low frequencies or for materials with high stiffness, the GBL-CH model and the Biot- $\mathrm{CH}$ model behave quantitatively similarly. Nevertheless, the microscopic fluctuation may be significant for some materials with low stiffness at high frequencies, particularly in cases where the effective solid density plays an important role e.g. in the case of resonance.

\section{Macroscopic sound absorption test}

In the previous section, the effective parameters obtained from the microscopic RVE in Fig. 2 have been studied and compared with semi-phenomenological models. The results partially agree qualitatively but a further investigation is still required for evaluation. In this section, the homogenization models are evaluated by following the same method as in the authors' previous work [49], where a macroscopic sound absorption set-up of a 2-cm porous layer shown in Fig. 7 was 
(a)

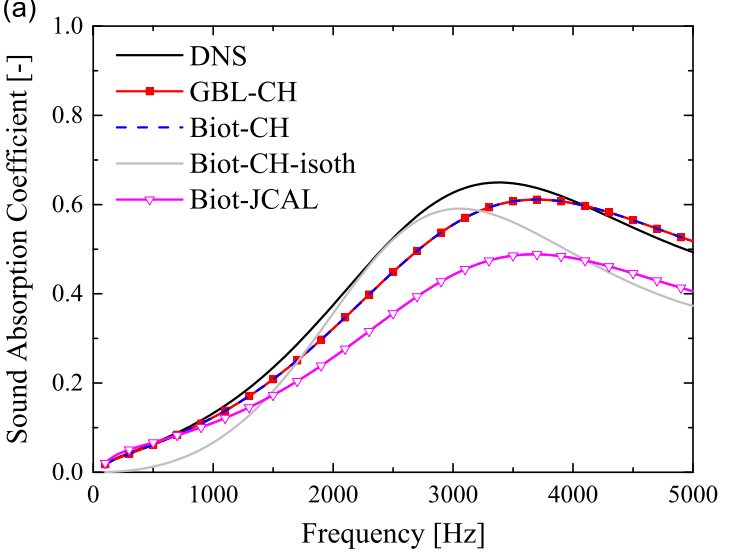

$\mathrm{Al}$

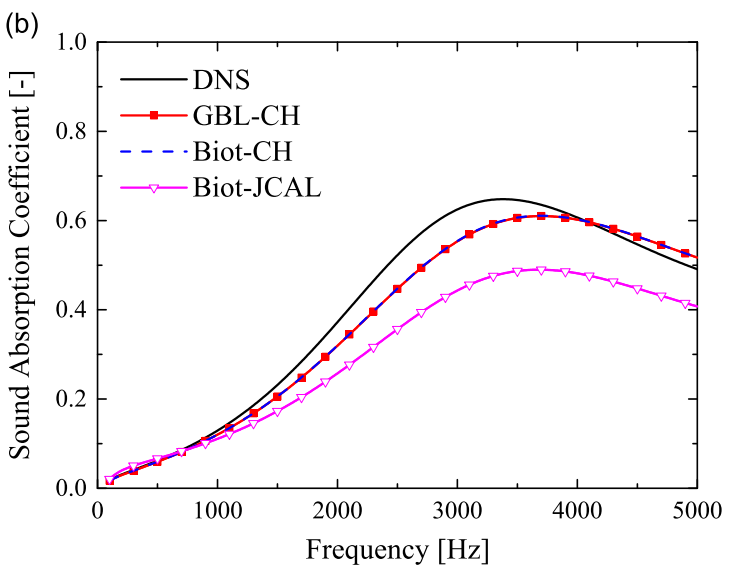

PU A

Fig. 8. Sound absorption coefficients of $\mathrm{Al}$ and PU A

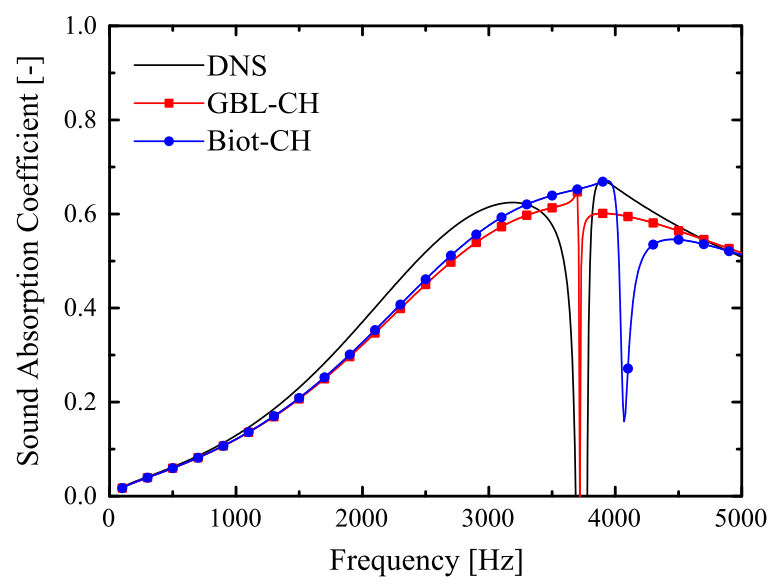

Fig. 9. Sound absorption coefficients of the simulations for PU B.

simulated and the result was compared with DNS containing the fully detailed microstructure corresponding to the RVE in Fig. 2.

The normal incident sound absorption coefficient is calculated by

$$
\alpha=1-\left|\frac{p_{\text {ref }}}{p_{\text {inc }}}\right|^{2},
$$

with the incident wave $p_{\text {inc }}=1$ Pa on the air-porous interface and the reflective wave $p_{\text {ref }}=p-p_{\text {inc }}$ where $p$ is the pressure on the interface. The sound absorption coefficient in the DNS is the average over the air-solid and the air-fluid interface. The results of $\mathrm{Al}$ and PU A are plotted in Fig. 8. Here, the isothermal result and the Biot-JCAL model are also included for comparison. The stiffness tensors ${ }^{4} \boldsymbol{C}^{s}$ are the same between the GBL-CH model and the Biot-JCAL model, because the difference between Fig. 8(a) and (b) is small: the relative difference between the DNS results is about 4 percent at $100 \mathrm{~Hz}$ and decreases to 0.4 percent at $5000 \mathrm{~Hz}$; the difference for the GBL-CH model is about 4 percent at $100 \mathrm{~Hz}$ and decreases to around 0.2 percent at $5000 \mathrm{~Hz}$. At low frequencies, due to the thermal dissipation, the non-isothermal results are higher than the isothermal result (the gray curve). At high frequencies, the influences of the thermal properties of the solid are less important and only the solid stiffness has an effect. The small difference at high frequencies between the results of two materials implies that the solid stiffness has a limited influence on the sound absorption performance for both $\mathrm{Al}$ and PU A.

As shown in Fig. 8, the sound absorption coefficients predicted by the GBL-CH model and the Biot-CH model are almost identical (the maximum relative difference is smaller than 0.002 percent for $\mathrm{Al}$ and 0.1 percent for PU A). Moreover, the results are lower than the DNS when the frequency is lower than $3000 \mathrm{~Hz}$. For a frequency between $800 \mathrm{~Hz}$ and $3000 \mathrm{~Hz}$, the prediction of the GBL-CH model is closer to the DNS than the Biot-JCAL model and improves with increasing frequency. When the frequency becomes higher than $3000 \mathrm{~Hz}$, the sound absorption coefficients are decreasing and the peaks of the GBL-CH model and the Biot-JCAL model both occur at higher frequencies than the DNS. The difference between the GBL-CH model and the Biot-JCAL model can be explained by the differences of the effective parameters discussed in the previous 


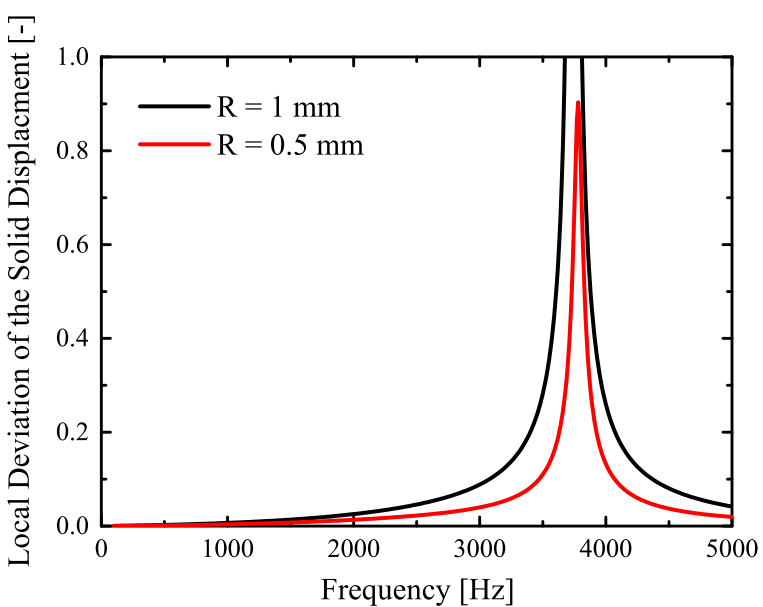

Fig. 10. Local deviation of the solid displacement in the DNS normalized by the characteristic macroscopic solid deformation $\epsilon_{M}^{*}$.

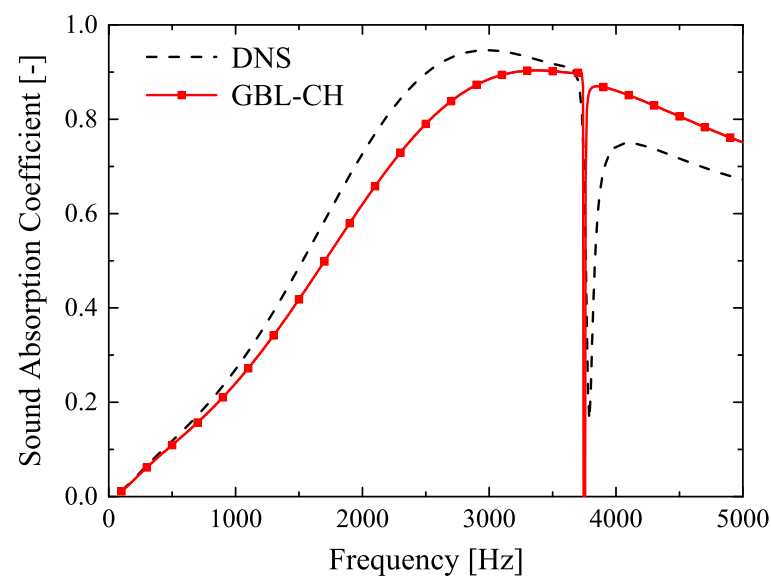

Fig. 11. Sound absorption coefficients of the 2-cm porous layer obtained from the DNS and the GBL-CH model for PU B, when the RVE is scaled down to $0.5 \mathrm{~mm}$.

section: the Biot-JCAL model provides a smaller magnitude and a shorter relaxation time for the effective fluid density and $R$ when the frequency is higher than around $1500 \mathrm{~Hz}$. This suggests that there is less dissipation in the Biot-JCAL model than in the GBL-CH model in this frequency range.

As discussed above, for materials with a high stiffness such as $\mathrm{Al}$ and PU A, the solid properties have a limited influence and the effective fluid properties determine the sound absorption performance. In this case, the behavior of the GBL-CH model is quite close to the Biot-CH model. Fig. 9 plots the sound absorption coefficients for PU B, showing that the resonance frequencies predicted by the two models are different: compared with the DNS, the GBL-CH model improves the resonance behavior relative to the Biot- $\mathrm{CH}$ model. This is because the microscopic fluctuation of the solid that is ignored in the Biot- $\mathrm{CH}$ model influences the resonance behavior. As shown in Fig. 4(a), the microscopic fluctuation of the solid increases the dynamic mass which reduces the resonance frequency. Note that the coefficient calculated by Eq. (47) cannot describe the absorbed energy correctly when resonance occurs, because the energy is mainly absorbed by the solid skeleton at the resonance frequency.

The difference between the DNS and the GBL-CH model is due to the boundary conditions adopted in the homogenization approach, which has been investigated by studying the boundary viscous stress and the scale separation principle in [49]. Particularly, the scale separation principle becomes less satisfied at increasing frequencies. Furthermore, Fig. 9 shows that the difference around the resonance frequency is large. Therefore, the scale separation principle is further analysed by following the method in [49]. The 2nd-derivative of the solid displacement that is averaged over the cross section perpendicular to the $x$ direction can be used to describe how the solid displacement in the DNS deviates from the periodicity assumption. This quantity is referred to as the local deviation and is plotted in Fig. 10. Here, it is normalized by the characteristic macroscopic solid deformation $\epsilon_{M}^{*}=\mid p_{\text {inc }} / E^{f r}$ (the estimated Young's modulus of the porous material $E^{f r}$ is calculated by Eq. (5-3) in [53]). The local deviation around the resonance frequency is considerably larger than at other frequencies, showing that periodicity is not well satisfied around the resonance frequency compared to other frequencies. 
Furthermore, when the size of the RVE is scaled down to $0.5 \mathrm{~mm}$, the local deviation becomes smaller and an improved performance of the GBL-CH model can be found in Fig. 11.

\section{Conclusions}

Acoustic problems of porous materials were investigated by using an extended homogenization method proposed in this paper. Based on the scale separation principle, the multiscale problem was divided into two separate problems at different scales: the scale of the macroscopic problem is controlled by the macroscopic solid displacement and fluid pressure; the scale of the microscopic RVE is described by linearized equilibrium equations and linear constitutive laws. The homogenization framework was built by applying a periodic solid displacement, a prescribed fluid traction and a periodic thermal flux on the boundary of the microscopic RVE. The micro-to-macro scale transition relations are obtained based on two-scale energy consistency.

The GBL-CH model was obtained by considering the linearity of the microscopic problem: the solid external force and the fluid displacement can be linearly expressed in terms of the solid displacement and fluid pressure gradient; the solid stress and the fluid volumetric change are related to the solid deformation and the fluid pressure. In this homogenization model, the required effective parameters are obtained through numerical simulations of the microscopic RVE. By ignoring the microscopic fluctuation terms, the Biot- $\mathrm{CH}$ model corresponding to Biot's poroelastic theory can be recovered from the GBL$\mathrm{CH}$ model. By simplifying the dynamic densities to being equal to the corresponding volume fractions, the GBL-CH model reduces to the Biot- $\mathrm{CH}$ model.

The effective parameters in the two models were discussed based on the simulations of an idealized cubic RVE. By considering the non-uniform temperature field, the thermal dissipation is involved in the effective modulus of the fluid and determines the sound absorption performance at low frequencies. It was found that the solid properties have limited effects on the effective fluid properties in the two models. However, the GBL-CH model shows that the solid can have a significant influence on the effective solid density, particularly in the high frequency range. This effect cannot be captured by the Biot$\mathrm{CH}$ model because it is mainly governed by the microscopic solid fluctuation field. Furthermore, a numerical simulation of a macroscopic sound absorption experiment on a porous material with the idealized cubic microstructure was simulated by using both models. A DNS result was used as a reference and the Biot-JCAL model was used for comparison purposes. By comparing the sound absorption coefficients, the GBL-CH model and the Biot-CH model outperform the Biot-JCAL model for the studied porous materials. It is also shown that the GBL-CH model gives a better prediction of the resonance frequency, which is affected by the microscopic fluctuation of the solid.

To summarize, the proposed novel homogenization approach has been successfully applied to acoustic porous materials. This new homogenization model can be considered as an extension of Biot's poroelastic theory, with incorporation of microscopic viscous-thermal effects. At low frequencies or for materials with a high stiffness, the performance of this model is almost the same as that of Biot's model. However, by comparing with the DNS, this new model gives a better prediction of the dynamic density of the solid affected by the microscopic fluctuation of the solid displacement.

\section{Acknowledgments}

This research was supported by the Dutch Technology Foundation STW, applied science division of NWO, and the Technology Program of the Ministry of Economic Affairs (under Grant number 10811).

\section{References}

[1] C. Zwikker, C.W. Kosten, Sound Absorbing Materials, Elsevier Publishing Company, New York, 1949.

[2] D.L. Johnson, J. Koplik, R. Dashen, Theory of dynamic permeability and tortuosity in fluid-saturated porous media, Journal of Fluid Mechanics 176 (1987) 379-402.

[3] Y. Champoux, J. Allard, Dynamic tortuosity and bulk modulus in air-saturated porous media, Journal of Applied Physics 70 (4) (1991) $1975-1979$.

[4] D. Lafarge, P. Lemarinier, J.F. Allard, V. Tarnow, Dynamic compressibility of air in porous structures at audible frequencies, The Journal of the Acoustical Society of America 102 (4) (1997) 1995-2006.

[5] J.F. Allard, B. Castagnède, M. Henry, W. Lauriks, Evaluation of tortuosity in acoustic porous materials saturated by air, Review of Scientific Instruments 65 (3) (1994) 754-755

[6] P. Leclaire, L. Kelders, W. Lauriks, M. Melon, N. Brown, B. Castagnède, Determination of the viscous and thermal characteristic lengths of plastic foams by ultrasonic measurements in helium and air, Journal of Applied Physics 80 (4) (1996) 2009-2012.

[7] R. Panneton, X. Olny, Acoustical determination of the parameters governing viscous dissipation in porous media, The Journal of the Acoustical Society of America 119 (4) (2006) 2027-2040.

[8] X. Olny, R. Panneton, Acoustical determination of the parameters governing thermal dissipation in porous media, The Journal of the Acoustical Society of America 123 (2) (2008) 814-824.

[9] D. Oliva, V. Hongisto, Sound absorption of porous materials-accuracy of prediction methods, Applied Acoustics 74 (12) (2013) $1473-1479$.

[10] O. Doutres, Y. Salissou, N. Atalla, R. Panneton, Evaluation of the acoustic and non-acoustic properties of sound absorbing materials using a threemicrophone impedance tube, Applied Acoustics 71 (6) (2010) 506-509.

[11] C. Perrot, F. Chevillotte, M. Tan Hoang, G. Bonnet, F.-X. Bécot, L. Gautron, A. Duval, Microstructure, transport, and acoustic properties of open-cell foam samples: experiments and three-dimensional numerical simulations, Journal of Applied Physics 111 (1) (2012) 014911. 
[12] F. Chevillotte, C. Perrot, E. Guillon, A direct link between microstructure and acoustical macro-behavior of real double porosity foams, The Journal of the Acoustical Society of America 134 (6) (2013) 4681-4690.

[13] O. Doutres, N. Atalla, K. Dong, A semi-phenomenological model to predict the acoustic behavior of fully and partially reticulated polyurethane foams, Journal of Applied Physics 113 (5) (2013) 054901.

[14] M. Tan Hoang, C. Perrot, Identifying local characteristic lengths governing sound wave properties in solid foams, Journal of Applied Physics 113 (8) (2013) 084905

[15] J. Auriault, L. Borne, R. Chambon, Dynamics of porous saturated media, checking of the generalized law of Darcy, The Journal of the Acoustical Society of America 77 (5) (1985) 1641-1650.

[16] C. Boutin, C. Geindreau, Estimates and bounds of dynamic permeability of granular media, The Journal of the Acoustical Society of America 124 (6) (2008) 3576-3593.

[17] C. Boutin, Acoustics of porous media with inner resonators, The Journal of the Acoustical Society of America 134 (6) (2013) 4717-4729.

[18] C. Boutin, F.X. Becot, Theory and experiments on poro-acoustics with inner resonators, Wave Motion 54 (2015) 76-99.

[19] D. Lafarge, N. Nemati, Nonlocal maxwellian theory of sound propagation in fluid-saturated rigid-framed porous media, Wave Motion 50 (6) (2013) $1016-1035$

[20] P. Göransson, Tailored acoustic and vibrational damping in porous solids-engineering performance in aerospace applications, Aerospace Science and Technology 12 (1) (2008) 26-41. aircraft noise reduction.

[21] N. Kino, G. Nakano, Y. Suzuki, Non-acoustical and acoustical properties of reticulated and partially reticulated polyurethane foams, Applied Acoustics 73 (2) (2012) 95-108.

[22] M.T. Hoang, G. Bonnet, H. Tuan Luu, C. Perrot, Linear elastic properties derivation from microstructures representative of transport parameters, The Journal of the Acoustical Society of America 135 (6) (2014) 3172-3185.

[23] M.A. Biot, Theory of propagation of elastic waves in a fluid-saturated porous solid. I. low-frequency range, The Journal of the Acoustical Society of America 28 (2) (1956) 168-178.

[24] M.A. Biot, Theory of propagation of elastic waves in a fluid-saturated porous solid. II. higher-frequency range, The Journal of the Acoustical Society of America 28 (2) (1956) 179-191.

[25] M.A. Biot, Mechanics of deformation and acoustic propagation in porous media, Journal of Applied Physics 33 (4) (1962) $1482-1498$.

[26] M.A. Biot, Generalized theory of acoustic propagation in porous dissipative media, The Journal of the Acoustical Society of America 34 (9A) (1962) $1254-1264$.

[27] J.M. Carcione, Wave propagation in anisotropic, saturated porous media: plane-wave theory and numerical simulation, The Journal of the Acoustical Society of America 99 (5) (1996) 2655-2666.

[28] A.-D. Cheng, Material coefficients of anisotropic poroelasticity, International Journal of Rock Mechanics and Mining Sciences 34 (2) (1997) 199-205.

[29] Y.J. Kang, J.S. Bolton, A finite element model for sound transmission through foam-lined double-panel structures, The Journal of the Acoustical Society of America 99 (5) (1996) 2755-2765.

[30] R. Panneton, N. Atalla, An efficient finite element scheme for solving the three-dimensional poroelasticity problem in acoustics, The Journal of the Acoustical Society of America 101 (6) (1997) 3287-3298.

[31] N. Atalla, R. Panneton, P. Debergue, A mixed displacement-pressure formulation for poroelastic materials, The Journal of the Acoustical Society of America 104 (3) (1998) 1444-1452.

[32] O. Dazel, B. Brouard, C. Depollier, S. Griffiths, An alternative Biots displacement formulation for porous materials, The Journal of the Acoustical Society of America 121 (6) (2007) 3509-3516.

[33] F.-X. Bécot, L. Jaouen, An alternative Biot's formulation for dissipative porous media with skeleton deformation, The Journal of the Acoustical Society of America 134 (6) (2013) 4801-4807.

[34] M.A. Biot, D.G. Willis, The elastic coefficients of the theory of consolidation, Journal of Applied Mechanics (1957) 594-601.

[35] P. Göransson, Acoustic and vibrational damping in porous solids, Philosophical Transactions of the Royal Society of London A: Mathematical, Physical and Engineering Sciences 364 (1838) (2006) 89-108.

[36] J.F. Allard, N. Atalla, Propagation of Sound in Porous Media: Modelling Sound Absorbing Materials, 2nd ed., John Wiley and Sons Ltd, United Kingdom, 2009.

[37] P. Khurana, L. Boeckx, W. Lauriks, P. Leclaire, O. Dazel, J.F. Allard, A description of transversely isotropic sound absorbing porous materials by transfer matrices, The Journal of the Acoustical Society of America 125 (2) (2009) 915-921, http://dx.doi.org/10.1121/1.3035840. URL http://scitation.aip.org/ content/asa/journal/jasa/125/2/10.1121/1.3035840.

[38] E. Lind-Nordgren, P. Göransson, Optimising open porous foam for acoustical and vibrational performance, Journal of Sound and Vibration 329 (7) (2010) $753-767$.

[39] B. Nennig, M.B. Tahar, E. Perrey-Debain, A displacement-pressure finite element formulation for analyzing the sound transmission in ducted shear flows with finite poroelastic lining, The Journal of the Acoustical Society of America 130 (1) (2011) 42-51.

[40] S.R. Pride, A.F. Gangi, F.D. Morgan, Deriving the equations of motion for porous isotropic media, The Journal of the Acoustical Society of America 92 (6) (1992) 3278-3290.

[41] K. Wilmanski, A few remarks on Biot's model and linear acoustics of poroelastic saturated materials, Soil Dynamics and Earthquake Engineering 26 (67) (2006) 509-536.

[42] E. Sanchez-Palencia, Non-Homogeneous Media and Vibration Theory, Springer, Berlin, Heidelberg, Germany, 1980.

[43] R. Burridge, J.B. Keller, Poroelasticity equations derived from microstructure, The Journal of the Acoustical Society of America 70 (4) (1981) $1140-1146$.

[44] J. Auriault, Heterogeneous medium. is an equivalent macroscopic description possible? International Journal of Engineering Science 29 (7) (1991) $785-795$.

[45] T. Yamamoto, S. Maruyama, K. Terada, K. Izui, S. Nishiwaki, A generalized macroscopic model for sound-absorbing poroelastic media using the homogenization method, Computer Methods in Applied Mechanics and Engineering 200 (1-4) (2011) $251-264$.

[46] O. Coussy, L. Dormieux, E. Detournay, From mixture theory to Biot's approach for porous media, International Journal of Solids and Structures 35 (3435) (1998) 4619-4635.

[47] M. Schraad, F. Harlow, A multi-field approach to modeling the dynamic response of cellular materials, International Journal of Mechanical Sciences 48 (1) (2006) 85-106.

[48] S.C. Cowin, L. Cardoso, Mixture theory-based poroelasticity as a model of interstitial tissue growth, Mechanics of Materials 44 (2012) $47-57$.

[49] K. Gao, J.A.W. van Dommelen, P. Göransson, M.G.D. Geers, A homogenization approach for characterization of the fluid-solid coupling parameters in Biot's equations for acoustic poroelastic materials, Journal of Sound and Vibration 351 (2015) 251-267.

[50] L.D. Landau, E.M. Lifshitz, Fluid Mechanics, 2nd ed., Course of Theoretical Physics, vol. 6, Pergamon Press, Oxford, United Kingdom, 1987.

[51] Polyurethane (PUR) plastic. URL 〈http://plastics.ulprospector.com/generics/45/polyurethane-pur (accessed: 01-04-2015).

[52] The engineering toolbox. URL 〈http://www.engineeringtoolbox.com/ $>$ (accessed: 01-04-2015).

[53] L.J. Gibson, M.F. Ashby, Cellular Solids-Structure and Properties, 2nd ed., Cambridge University Press, Cambridge, United Kingdom, 1999. 